\title{
DEMONSTRATION OF ULTRA HI-FI (UHF) METHODS
}

\author{
Rodger W. Dyson \\ NASA Glenn Research Center \\ 21000 Brookpark Road \\ Cleveland, $\mathrm{OH} 44135$ \\ Rodger.W.Dyson@nasa.gov \\ 216-433-3918 (FAX) \\ 216-433-9083 (VOICE)
}

\begin{abstract}
Computational aero-acoustics (CAA) requires efficient, high-resolution simulation tools. Most current techniques utilize finite-difference approaches because high order accuracy is considered too difficult or expensive to achieve with finite volume or finite element methods. However, a novel finite volume approach (Ultra HI-FI or UHF) which utilizes Hermite fluxes is presented which can achieve both arbitrary accuracy and fidelity in space and time. The technique can be applied to unstructured grids with some loss of fidelity or with multi-block structured grids for maximum efficiency and resolution. In either paradigm, it is possible to resolve ultra-short waves $(<2$ PPW). This is demonstrated here by solving the $4{ }^{\text {th }}$ CAA workshop Category 1 Problem 1 .
\end{abstract}

\section{INTRODUCTION}

Simulating sound generation, propagation, and reflection is notoriously demanding because of the wide range of scales. For the past decade many of the successful CAA applications have been based upon finite difference methodologies because it was not clear how to achieve high numerical resolution and efficiency with finite volume and finite element approaches. Commercial computational fluid dynamics developers long ago settled on finite volume approaches for steady calculations because shocks and complex geometry are more naturally dealt with (ref. 1). Unfortunately, the low resolution and efficiency of CFD required new approaches - hence, the development of finite-difference based schemes with wide and/or Padé stencils (refs. 2 to 3). In (ref. 3) and later (ref. 4) the advantage of a staggered grid was demonstrated and it was suggested finite volume techniques could naturally utilize this property. The development of higher order finite volume and finite element schemes has quietly progressed (refs. 4 to 11). And more recently, very high order finite volume approaches have been developed (refs. 12 to 14). These recent developments demonstrate the feasibility and value of high order, low dissipation, low dispersion finite volume formulations. Unfortunately, none of these approaches can achieve both arbitrary accuracy and fidelity in both space and time for nonlinear aeroacoustic flows in complex geometries. As shown in Figure 1, conventional techniques require about 6 points per wavelength (PPW) for reasonable resolution and a lack of resolution directly affects the overall efficiency (ref. 15). This can make a significant difference in the tractability of simulating complex engineering devices.
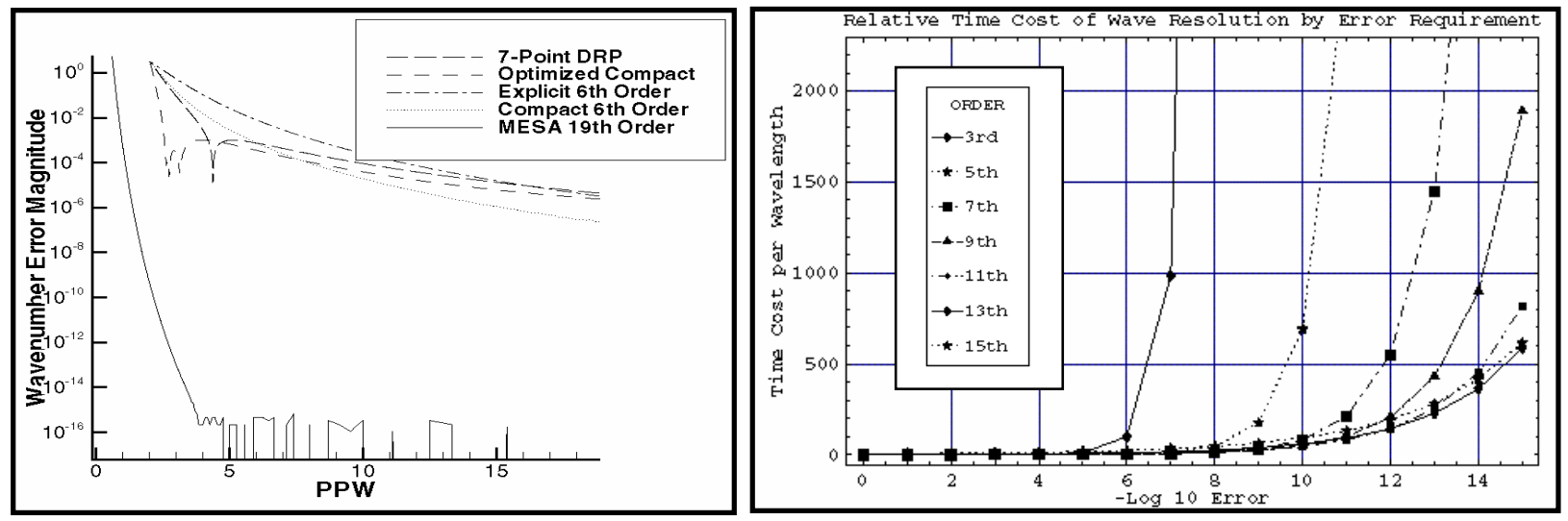

Figure 1 - Resolution and Efficiency Comparison 
The desire to pursue a high resolution finite volume approach in turbo-machinery aero-acoustics is in part driven by the need to simulate the following:

- Mild shocks near transonic blade tips

- Sharp blade leading edges with steep flow gradients

- Sharp blade trailing edges to properly account for the Kutta condition

- Vortical, entropic, and acoustic wave propagation over approximately 10 wavelengths

It is also due in part by the need to maintain the following properties:

- Flow conservation in difficult areas such as blade leading edge and trailing edge (see Figure 2)

- Isotropic shock and wave speed everywhere in the machinery

- Nonlinear effects of blade curvature and active control surfaces

- Isentropic (for inviscid flows) flow along solid boundaries

Finite difference approaches have the following impediments to meeting the above needs:

- Structured, Overset, or Cartesian grids are either difficult to apply to complex geometries or grid singularities, as shown in Figure 3, occur as in the case of multi-block structured solvers

- Determining derivatives with wide stencils or compact schemes are undefined at shocks resulting in instabilities, shock smearing, etc.

- Corners pose difficulties (i.e. are multiply defined at wall/inflow and nonreflecting conditions require special treatment there)

- Spatial filtering/damping requires one-sided derivatives at boundaries which effectively changes the dispersion relations

- First order grid singularity noise sources arise from both multi-block curvilinear grids and sharp edged complex geometry

These considerations are addressed by using Ultra Hermite Integration, Flux, and Interpolation (Ultra HI-FI or UHF) methods. The term "Ultra" refers to the most salient characteristic of these methods which is they can resolve ultra-short waves ( $<2$ PPW) (ref. 16). This level of fidelity was first achieved with a finite difference HermiteTaylor approach (ref. 17). In a similar vein, UHF methods use Cauchy-Kowalewski-Taylor time advance, and the spatial derivatives of the cell averages are carried on the grid; And Hermite fluxes are calculated and used to conservatively time advance all derivatives of the cell averages (ref. 6). This can be formulated with unstructured grids with upwinding (ref. 6); It can also be formulated in a centered staggered grid as by Chang/Van Leer (refs. 18 to 20). But the most efficient formulation is structured multi-block curvilinear because the geometry is implicitly represented to higher fidelity as higher derivatives of the metric terms are naturally included from differentiating the strong conservation of the governing equations. For example, the variation of the coordinate transformation first derivatives (metric term, $\eta_{\mathrm{x}}$ ) near a typical leading edge is show in figure 4 .

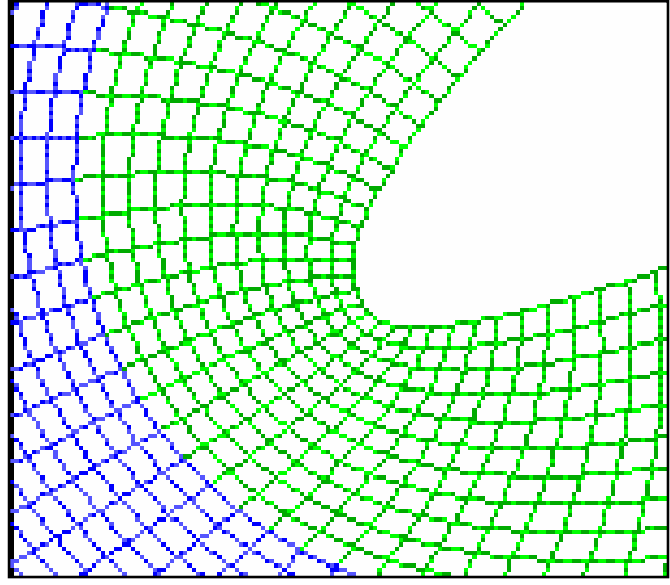

Figure 2 - Flux Conservation

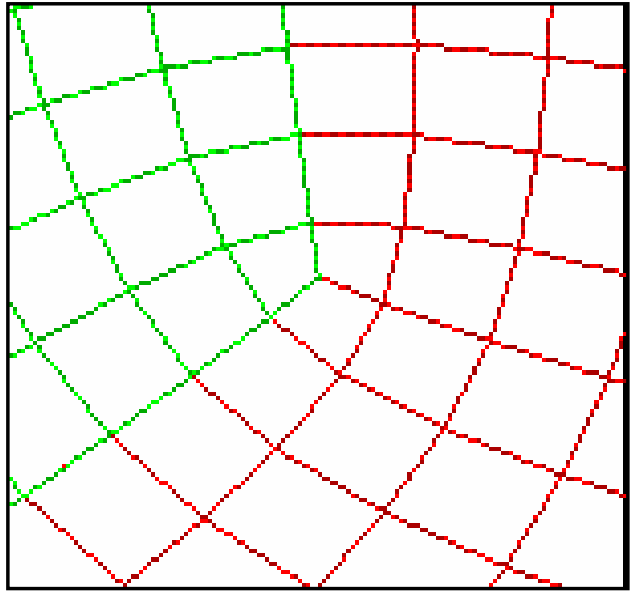

Figure 3 --Grid Singularities 


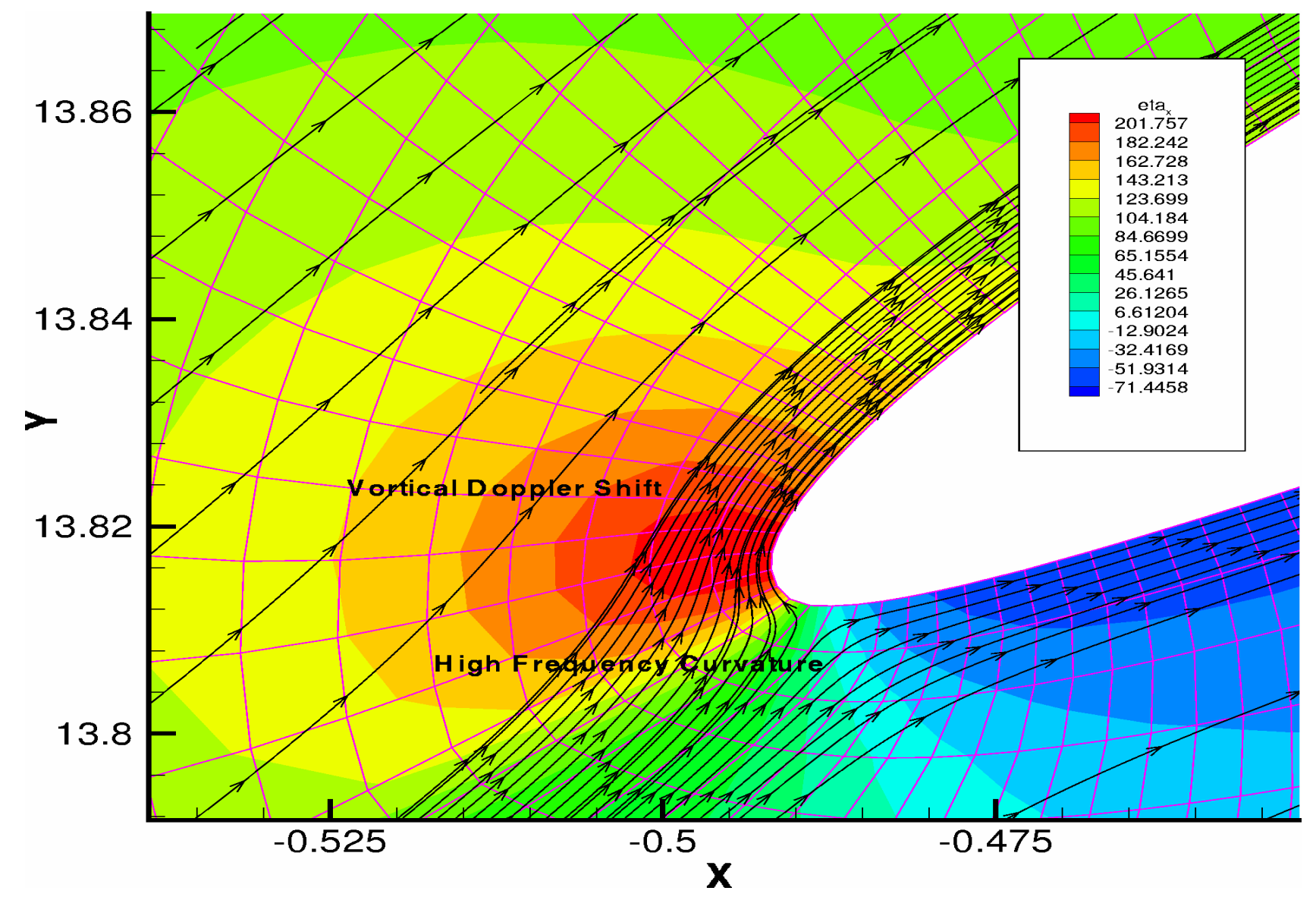

Figure 4 -- Spatial variation of curvilinear metric terms

\section{NUMERICAL APPROACH}

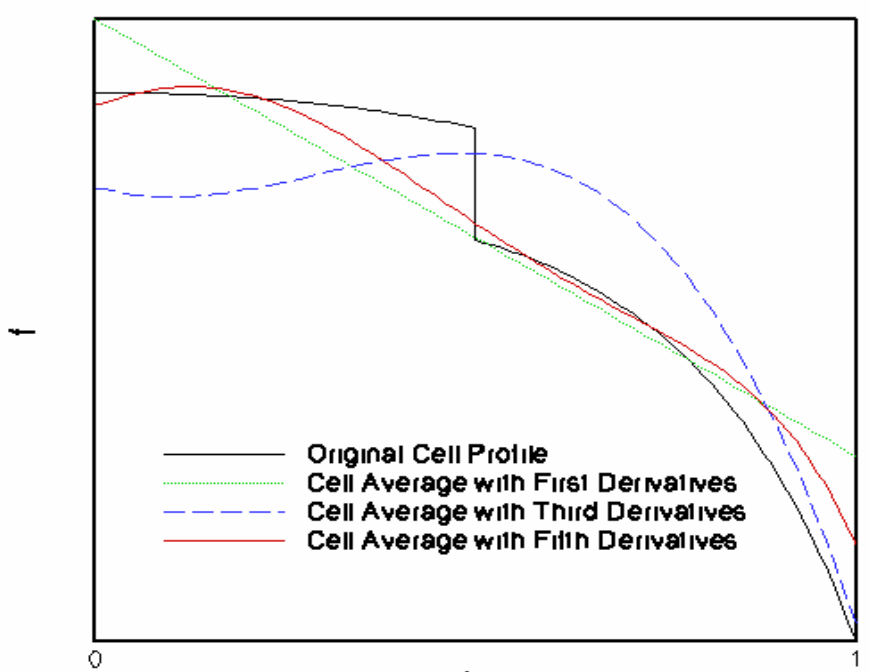

Figure 5 - Reconstruction of Shock
The key idea is to include spatial derivatives of the cell averages on the grid (ref. 6) which as shown in Figure 5 provide better shock fidelity as more derivative information is used in the reconstruction. The spatial derivative of the cell average is still defined at shocks which addresses the common criticism of high order finite difference methods. For the one-dimensional advection equation:

$$
\boldsymbol{u}_{t}+\boldsymbol{u}_{\boldsymbol{x}}=\mathbf{0}
$$

the following cell averages are stored on the grid:

$$
\begin{aligned}
\bar{u} & =\left(\frac{1}{\Delta x}\right) \int u d x \quad \bar{u}_{x}=\left(\frac{1}{\Delta x}\right) \int u_{x} d x \\
\bar{u}_{x x} & =\left(\frac{1}{\Delta x}\right) \int u_{x x} d x \quad \bar{u}_{x x x}=\left(\frac{1}{\Delta x}\right) \int u_{x x x} d x
\end{aligned}
$$

More derivatives can be added as well to provide the fidelity and efficiency as shown in Figure 1. 
The integral form of the governing equations (eq. 1) may be repeatedly differentiated:

$$
\begin{gathered}
\int u_{t} d V+\int u_{x} d V=\bar{u}_{t} \Delta x+u(j+1)-u(j)=0 \\
\int u_{t x} d V+\int u_{x x} d V=\bar{u}_{t x} \Delta x+u_{x}(j+1)-u_{x}(j)=0 \\
\int u_{t x x} d V+\int u_{x x x} d V=\bar{u}_{t x x} \Delta x+u_{x x}(j+1)-u_{x x}(j)=0 \\
\int u_{t x x x} d V+\int u_{x x x x} d V=\bar{u}_{t x x x} \Delta x+u_{x x x}(j+1)-u_{x x x}(j)=0
\end{gathered}
$$

For this simple case the flux is simply the conserved variable, u. We need to reconstruct the sampled or unaveraged variables from the known averaged variables to calculate the flux at $\mathrm{J}=0$ and $\mathrm{J}=1$ in Figure 6.

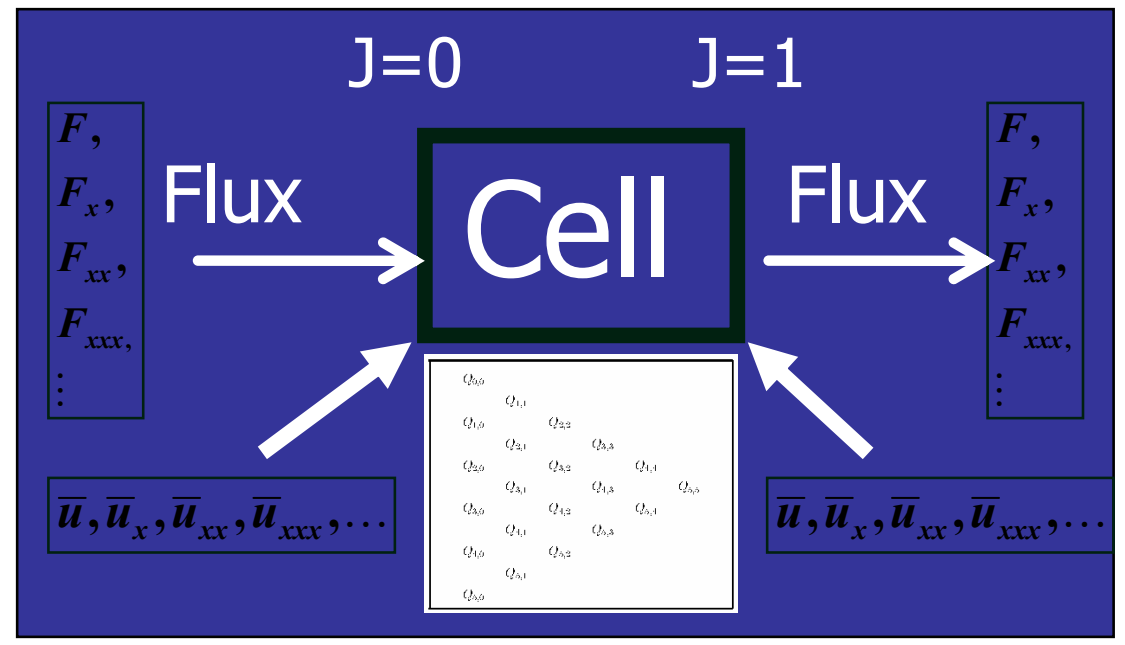

Figure 6- Basic Cell

This reconstruction can be accomplished in two ways, either by reconstruction via the primitive function (RP) (ref. 21) or by using constraints (ref. 22). For the structured curvilinear staggered UHF method one would use two cells for the reconstruction with the following constraints:

$$
\begin{gathered}
u(\xi)=\sum_{i=0}^{2 s+1} a_{i} \xi^{i} \\
\frac{1}{\Delta x} \int_{-\Delta x / 2}^{\Delta x / 2} \frac{\partial^{m} u(\xi)}{\partial x^{m}} d \xi=\frac{\partial^{m} \bar{u}_{j}}{\partial x^{m}} \\
\frac{1}{\Delta x} \int_{\Delta x / 2}^{3 \Delta x / 2} \frac{\partial^{m} u(\xi)}{\partial x^{m}} d \xi=\frac{\partial^{m} \bar{u}_{j}}{\partial x^{m}} \\
\text { with } \mathrm{m}=0 \text { up to s. }
\end{gathered}
$$

For very high order UHF methods this process is intractable because current computer algebra techniques cannot symbolically invert large matrices and in those cases the RP approach using Hermite divided differences is more practical. 
After calculating the fluxes with (eq. 4), the first time derivatives of the cell average spatial derivatives are found with (eq. 3). Next, the Cauchy-Kowalewski procedure can be applied as follows since from (eq. 1) we have:

$$
\begin{gathered}
\bar{u}_{t}=\bar{u}_{t}, \bar{u}_{t t}=-\bar{u}_{x t}, \bar{u}_{t t t}=\bar{u}_{x x t} \\
\bar{u}_{x t}=\bar{u}_{x t}, \bar{u}_{x t t}=-\bar{u}_{x x t}, \bar{u}_{x t t t}=\bar{u}_{x x x t} \\
\bar{u}_{x x t}=\overline{\boldsymbol{u}}_{x x t}, \overline{\boldsymbol{u}}_{x x t t}=-\overline{\boldsymbol{u}}_{x x x t}, \overline{\boldsymbol{u}}_{x x t t}=\overline{\boldsymbol{u}}_{x x x x t}
\end{gathered}
$$

This procedure can by performed efficiently with unrolled recursion (ref. 15). At this point we can time advance the cell average data with a simple Taylor series in time:

$$
\begin{gathered}
\bar{u}^{n+1}=\bar{u}^{n}+\sum_{k=1}^{\text {order }}\left(\frac{1}{k !}\right) \frac{\partial^{k} \bar{u}^{n}}{\partial t^{k}}(\Delta t)^{k} \\
\bar{u}_{x}^{n+1}=\bar{u}_{x}^{n}+\sum_{k=1}^{\text {order }}\left(\frac{1}{k !}\right) \frac{\partial^{k} \bar{u}_{x}^{n}}{\partial t^{k}}(\Delta t)^{k} \\
\bar{u}_{x x}^{n+1}=\bar{u}_{x x}^{n}+\sum_{k=1}^{\text {order }}\left(\frac{1}{k !}\right) \frac{\partial^{k} \bar{u}_{x x}^{n}}{\partial t^{k}}(\Delta t)^{k} \\
\bar{u}_{x x x}{ }^{n+1}=\bar{u}_{x x x}{ }^{n}+\sum_{k=1}^{\text {order }}\left(\frac{1}{k !}\right) \frac{\partial^{k} \bar{u}_{x x x}{ }^{n}}{\partial t^{k}}(\Delta t)^{k}
\end{gathered}
$$

The cell average data, $\overline{\boldsymbol{u}}^{n},\left(\overline{\boldsymbol{u}}^{n}\right)_{x},\left(\overline{\boldsymbol{u}}^{n}\right)_{x \boldsymbol{x}},\left(\overline{\boldsymbol{u}}^{n}\right)_{x x \boldsymbol{x}}, \ldots$. , at the $\mathrm{J}=1 / 2$ location in Figure 6 is simply interpolated using Hermite divided differences (ref. 15). Or, if the single cell unstructured UHF is used (ref. 6) then this information is already present.

After this process the following cell averaged data, $\bar{u}^{n+1},\left(\bar{u}^{n+1}\right)_{x},\left(\bar{u}^{n+1}\right)_{x x},\left(\bar{u}^{n+1}\right)_{x x x}, \ldots$, is known at $\mathrm{J}=1 / 2$ in Figure 6 . The process just described is repeated at the next time level resulting in a staggered, centered UHF method. If an unstaggered single cell reconstruction is used as in (ref. 6), then an upwinding dissipation term should be included for stability.

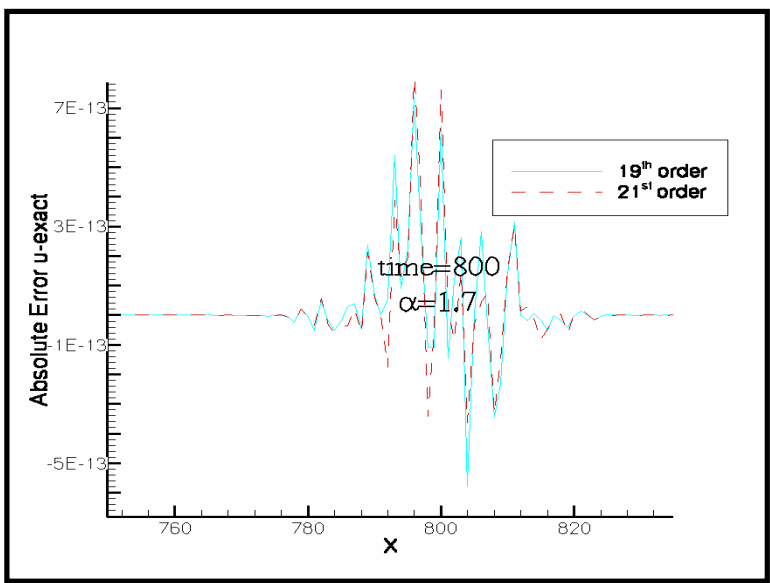

Figure 7 -- 3.69 PPW

\section{RESULTS}

The advection equation (1) is solved using the centered, staggered UHF procedure just described with the following initial condition:

$$
u(t=0)=[2+\cos (\alpha x)] \exp \left[-(\ln 2)(x / 10)^{2}\right](7)
$$

The results at time $=800$ are shown for $\alpha=1.7, \alpha=4.6$, and $\alpha=20$ in figs. 7 to 9 . Notice that Figures 8 and 9 show ultrashort wave resolution with at least 7 digits of accuracy. 


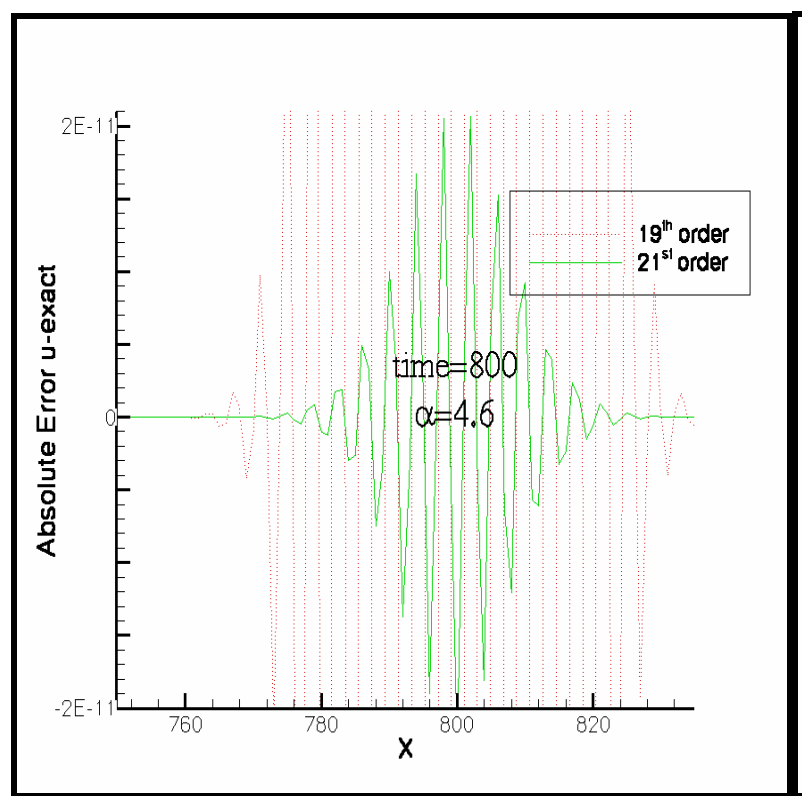

Figure 8-1.36 PPW

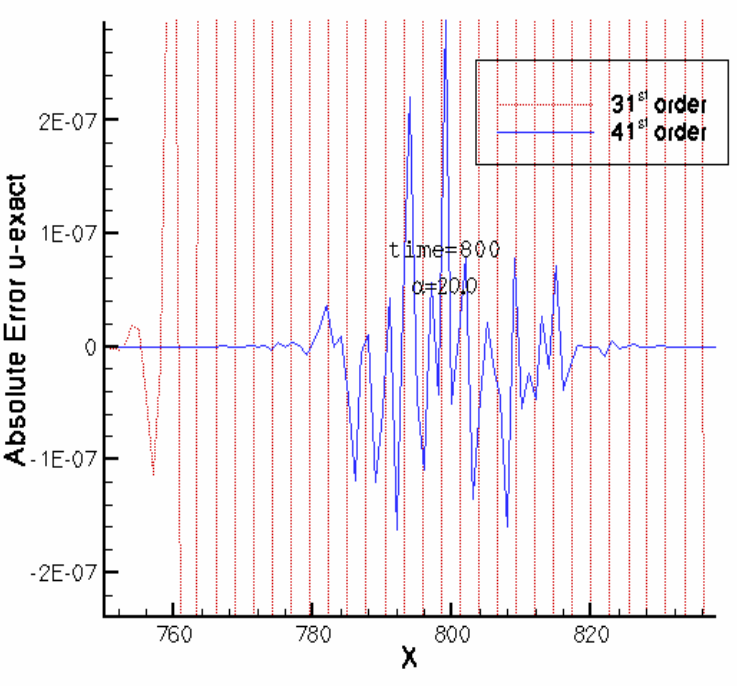

Figure 9 -- .314 PPW

This performance can be better understood by examining the UHF method's amplification factor as a function of wavenumber. Performing the usual Fourier linear modal analysis, assume a harmonic solution of the form in (1):

$$
\begin{gathered}
u(x, t)=A e^{i k(x-t)} \\
g_{\text {exact }}=\left|\frac{u^{n+1}}{u^{n}}\right|=1 \quad\left(g_{\text {exact }}\right)_{x}=\left|\frac{u_{x}^{n+1}}{u_{x}^{n}}\right|=1 \quad\left(g_{\text {exact }}\right)_{x x}=\left|\frac{u_{x x}{ }^{n+1}}{u_{x x}{ }^{n}}\right|=1
\end{gathered}
$$

We can immediately conclude that not only should the amplitude of all the waves remain unchanged, but that the amplitudes of the spatial derivatives of the waves should not change. By performing this analysis for $\mathrm{k}>\pi$, we find that UHF methods are indeed resolving the ultra-short waves as shown in Figure 10. Notice the addition of 3 more spatial derivatives clearly improves the fidelity beyond $\mathrm{k}>2 \pi$.

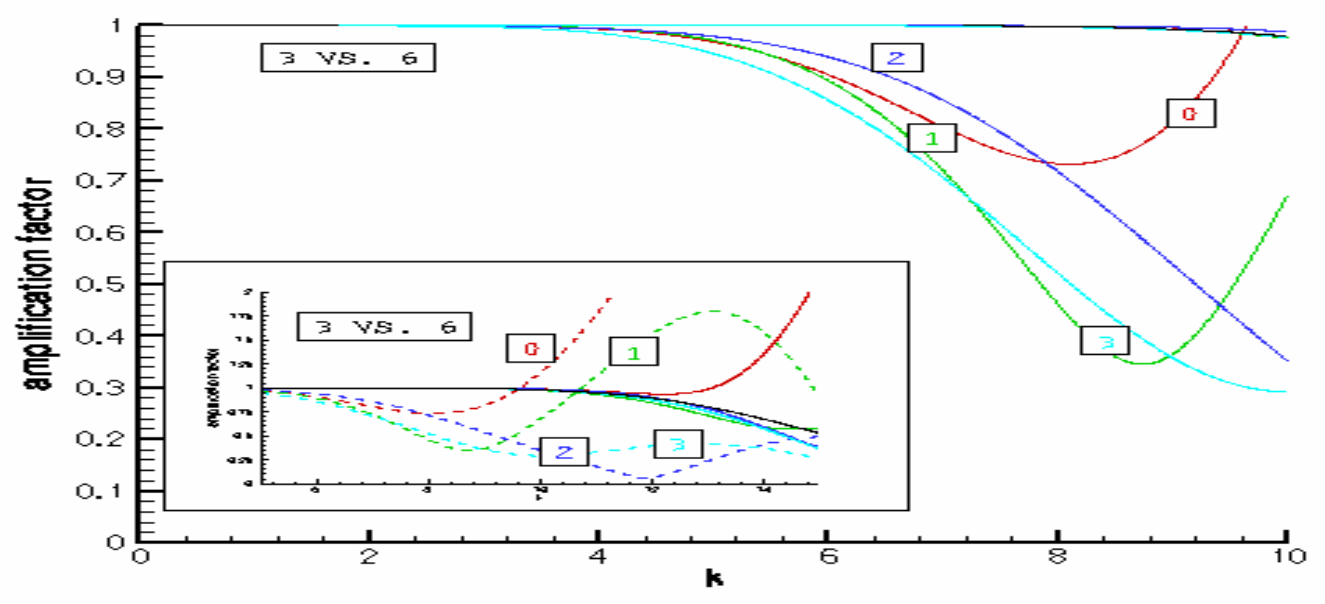

Figure 10 -- Fidelity Comparison of 3 and 6 spatial derivatives of the cell averages on the grid 


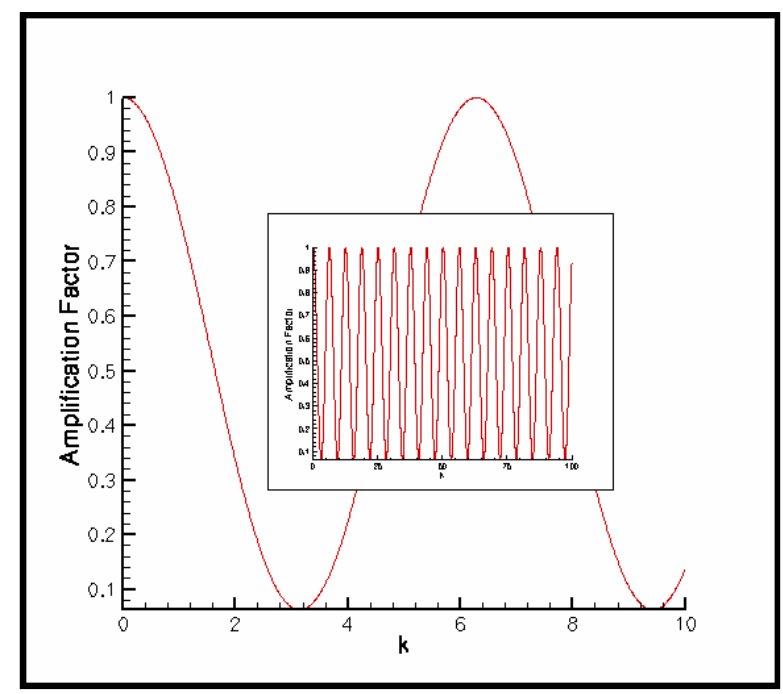

Figure 11 - No derivatives repeats

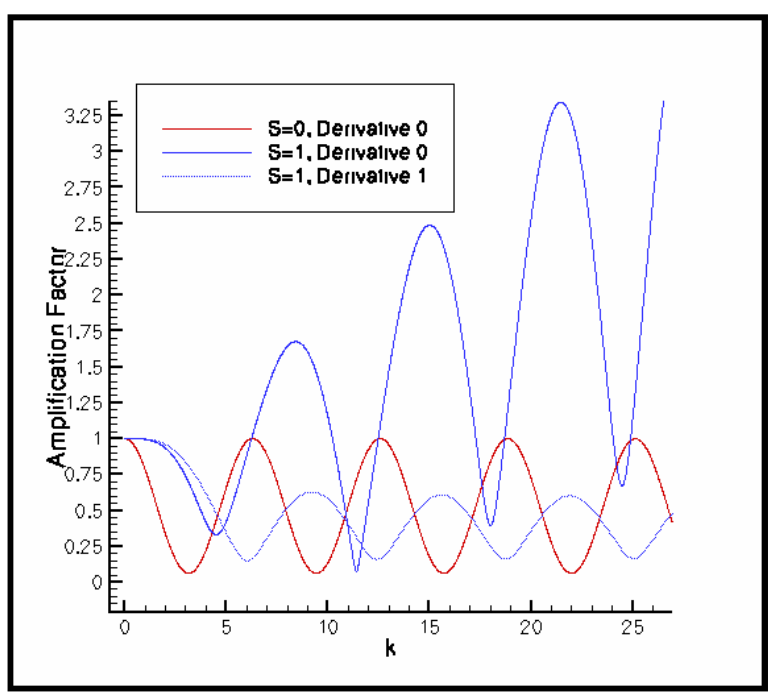

Figure 12 - Single derivative comparison

In Figure 11 we can see that the amplification factor merely repeats itself every $\mathbf{k}=\pi$ which is another way of observing ultra-short aliased waves are indistinguishable on the grid. Notice in Figure 12 that adding just one derivative to the calculation results in significant growth of the extremely short waves but in practice the method is stable because of the interaction of the damped spatial derivatives with the undamped portion of the ultra-short wave solution variables.

\section{CONCLUSION}

Much remains to be explored but it is clear that the ultra-short wave resolution implies significant efficiencies are attainable while keeping the desirable properties of finite volume formulations. New ultra-wave filters will need to be developed to fully utilize this level of fidelity (ref. 23). A discussion of boundary conditions is beyond the scope of the paper but the interested reader may find (ref. 24) helpful. The extension to Euler equations is laid out in (ref. 6) for unstructured single cell reconstruction and a demonstration of the multiblock structured two-cell reconstruction is being prepared. Space limitations preclude explaining more.

\section{ACKNOWLEDGEMENTS}

This work is the product of the education received from Dr. John Goodrich while I was his Ph.D. student and the practical training I received from Dr. Ray Hixon while he masterfully integrated many areas of CAA into a working code. 


\section{REFERENCES}

1. Blazek, J.; Computational Fluid Dynamics: Principles and Applications. Elsevier, New York, 2001

2. Tam, C.K.W, Webb, J.C.: Dispersion-Relation-Preserving Finite-Difference Schemes for Computational Acoustics, J. Comp. Phys., Vol. 107, No. 2, 1993, pp. 262-281

3. Lele, S.K. : Compact Finite Difference Schemes with Spectral-like Resolution, J. Comp. Physics, Vol. 103, 1992, pp. 16-42

4. Nagarajan, S., Lele, S.K., Ferziger, J.H.: A robust high-order compact method for large eddy simulation, J. Comp. Physics, Vol. 191, 2003, pp. 392-419

5. Shu, C.: High-order Finite Difference and Finite Volume WENO Schemes and Discontinuous Galerkin Methods for CFD, Int. J. CFD, Vol. 17, No. 2, 2003, pp. 107-118

6. Agarwal, R. K., and Halt, D.W. : A Compact High-order Unstructured Grids Method for the Solution of Euler equations, Int. J. for Num. Meth. In Fluids, Vol. 31, 1999, pp.121-147

7. Wang, G. and Sankar, L.N. : Prediction of Rotorcraft noise with a low-dispersion finite Volume Scheme", Vol. 38, No. 3, March 2000

8. Hernandez, J.A. : High-order finite volume schemes for the advection-diffusion equation, Int. J. Num. Meth. Eng., Vol. 53, 2002, pp. 1211-1234

9. Rezgui, A., Cinnella, P., Lerat, A. : Third-order accurate Finite Volume Schemes for Euler Computations on Curvilinear Meshes, Computers \& Fluids, Vol. 30, 2001, pp. 875-901

10. Nance, D.V., Viswanathan, K., Sankar, L.N.: Low-dispersion Finite Volume Scheme for Aeroacoustic Applications, AIAA J., Vol. 35, No. 2, Feb. 1997

11. Gaitonde, D., Shang, J.S.: High-order finite-volume schemes in wave propagation phenomena, AIAA Paper 96-2335, Plasmadynamics and Lasers Conference, June 17-20, 1996

12. Wang, Z.J. : Spectral (finite) volume method for conservation laws on unstructured grids: basic formulation, J. Comput. Phys., Vol. 178, No. 210, 2002

13. Wang, Z.J., Liu, Y. : Spectral (finite) volume method for conservation laws on unstructured grids II: extension to two-dimensional scalar equation, J. Comput. Phys., Vol. 179, 2002, pp. 665-697

14. Schwartzkopff, T., Munz, C.:Fast ADER: An Arbitrary High Order Scheme for Linear Acoustics, $4^{\text {th }}$ CAA Workshop, OAI, Cleveland, OH, Oct. 2003

15. Dyson, R.W.: Technique for Very High Order Nonlinear Simulation and Validation, J. Comp. Acoustics, Vol. 10, No. 2, 2002, pp. 211-229

16. Tam, C.K.W. : Numerical Methods in Computational Aeroacoustics Short Course, Breckenridge, Colorado, June 15-16, 2002

17. Dyson, R.W. and Goodrich, J.W.: Automated Approach to Very High-Order Aeroacoustic Computations, AIAA J., Vol. 39, No. 3, 2001, pp. 396-406

18. Chang, S.-C.: The Method of Space-Time Conservation Element and Solution Element - A New Approach for Solving the Navier-Stokes and Euler equations, J. Comp. Physics, Vol. 119, 1995, pp. 295-324

19. Van Leer, B.:Towards the Ultimate Conservative Difference Scheme IV. A New Approach to Numerical Convection, J. Comp. Physics, Vol. 23, 1977, pp. 276-299

20. Huynh, H.T.: Analysis and Improvement of Upwind and Centered Schemes on Quadrilateral and Triangular Meshes, AIAA Paper 2003-3541, $16^{\text {th }}$ AIAA Computational Fluid Dynamics Conference, June 23-26, 2003

21. Collela, P. and Woodward, P.R.: The Piecewise Parabolic Method (PPM) for Gas-Dynamical Simulations, J. Comp. Phys., Vol. 54, 1984, pp. 174-201

22. Lomax, H.; Pulliam, T.H., Zingg, D.W.: Fundamentals of Computational Fluid Dynamics, Springer-Verlag, 2001

23. Zhou, Y.C., Wei, G.W.: High resolution conjugate filters for the simulation of flow, J. Comp. Phys., Vol. 189, 2003, pp. 159-179

24. Dyson, R.W.; Hixon, R.: Towards Arbitrary Accuracy Inviscid Surface Boundary Conditions, AIAA-20022438, NASA-TM-2002/211583, 2002 